\section{FORMACIÓN CIUDADANA EN EL PROGRAMA DE PSICOLOGÍA DE LA UNIVERSIDAD NACIONAL ABIERTA Y A Distancia, CEAD IBAgué}

\author{
CITIZEN TRAINING IN THE PSYCHOLOGY PROGRAM \\ OF THE NATIONAL OPEN AND DISTANCE \\ UNIVERSITY, CEAD IBAGUÉ
}

Natalia Arbeláez Gallego*

José Julián Ñañez Rodríguez**
* Magíster en Educación de la Universidad Surcolombiana. Profesora Tiempo Completo de la Universidad Cooperativa de Colombia Sede Ibagué. nati.arbelaezg@gmail.com 0000-0003-1481-3304

** Doctor en Educación de la Universidad del Tolima. Profesor de la Universidad del Tolima.

jjnanezr@ut.edu.co 0000-0002-1221-7050

Cómo citar este artículo: Arbeláez, N. \& Ñañez, R. J. J. (2021). Formación ciudadana en el programa de Psicología de la Universidad Nacional Abierta y a Distancia, CEAD Ibagué. Revista PACA 11, pp. 67-89.
Aceptado: Octubre 19, 2021

Resumen: El presente artículo presenta la valoración y el aporte del programa de Psicología de la UNAD, CEAD Ibagué, en lo relacionado con la formación ciudadana de sus estudiantes, a través del análisis del Proyecto Académico Pedagógico Solidario (PAPS) y del Plan de Estudio del programa de Psicología de la UNAD. Los objetivos que movilizan esta apuesta pedagógica están orientados de manera general a valorar el aporte del programa de Psicología de la UNAD, CEAD Ibagué, en lo relacionado con la formación ciudadana de sus estudiantes. De manera específica se buscó analizar en el proyecto académico pedagógico solidario (PAPS) y en el Plan de Estudio del programa de Psicología de la UNAD, los aspectos relacionados con la formación ciudadana a los estudiantes; así mismo, describir los aportes a la formación ciudadana desde las actividades extracurriculares $\mathrm{y} / \mathrm{o}$ complementarias dirigidas a los/las estudiantes de Psicología, CEAD Ibagué y, finalmente, analizar las concepciones que poseen los / las estudiantes, docentes y egresados sobre la formación ciudadana que imparte el programa de Psicología de la UNAD - CEAD Ibagué. Metodológicamente es de enfoque mixto, con un alcance descriptivo desde el estudio de caso. Se establecieron también audiencias foco conformadas por la comunidad estudiantil del programa de Psicología del CEAD Ibagué, compuestas 
por una muestra de 18 estudiantes que se encuentren realizando sus prácticas profesionales. Así mismo, un grupo de cinco (5) docentes del programa de Psicología del CEAD Ibagué, quienes participaron en la formación de los estudiantes de prácticas profesionales; y otro grupo de cinco (5) egresados del programa de Psicología del CEAD Ibagué para el grupo focal. Por otro lado, los instrumentos aplicados fueron: la revisión documental, la encuesta y el grupo focal. Los datos se analizaron a través de la triangulación de información, con lo cual se estableció que la valoración significativa se expresó al evidenciarse un acoplamiento en el discurso de los docentes, estudiantes y egresados con las directrices de la UNAD, expresadas en diversos documentos como el PAPS y los lineamientos del programa de Psicología, pues en cada uno de estos componentes de análisis se tuvo en cuenta la formación ciudadana como manera de abordar y dimensionar las problemáticas sociales.

Palabras clave: formación ciudadana, ciudadanía, psicología, educación superior.

Abstract: This article presents the assessment and contribution of the Psychology program of the UNAD, CEAD Ibagué, in relation to the citizen training of its students, through the analysis of the Solidarity Pedagogical Academic Project (PAPS) and Study Plan of the program of Psychology of the UNAD. Methodologically it is of mixed approach, with a descriptive scope from the case study. It was also established, focus audiences formed by the student community of the Psychology program of CEAD Ibagué, composed of a sample of 18 students who are doing their professional practices. Likewise, a group of five (5) teachers from the Psychology program of the CEAD Ibagué who participated in the training of the internship students and another group of five (5) graduates of the Psychology program of the CEAD Ibagué for the focus group. On the other hand, the instruments applied were: the documentary review, the survey and the focus group. The data is analyzed through the triangulation of information. Therefore, it was established that the significant assessment was expressed when there was a link in the discourse of teachers, students and graduates with the guidelines of the UNAD expressed in various documents such as the PAPS and the guidelines of the Psychology program, because in each one of these components of analysis took into account citizen training as a way to address and dimension social problems.

Keywords: citizen training, citizenship, psychology, higher education.

\section{Introducción}

Nuestra sociedad ha buscado promover una educación que sea la expresión de los retos, capacidades y potencialidades que engendra un proyecto de 
nación incipiente en términos de identidad y sentidos de pertenencia, y como apuesta para reconfigurar las dinámicas, tensiones y vicisitudes del ser ciudadano desde la cotidianidad. En esta iniciativa ha cobrado un peso significativo la formación por competencias, la cual busca promover y garantizar la adquisición de unas competencias básicas que impulsen la formación integral; de modo que la participación política se manifieste de múltiples formas y en diversos contextos, siempre mediada por una actitud de vínculo afectivo y racional con sus iguales, pero también por una crítica permanente. Gracias a la crítica y a la organización social y política, lo público permite ir transformando el poder de la dominación en poder legítimo, con base, precisamente, en el "poder comunicativo" que se genera en los procesos de formación y cultura democrática (Hoyos, 2001).

Alvarado (1999) afirma que para construir la sociedad se debe construir primero un proyecto de vida, un proyecto educativo (no escolar) que "permita reflexionar sobre los problemas de la conciencia moral, la equidad y la inequidad, la búsqueda de la identidad, el sentido de la libertad y para esto se requiere que cada ciudadano adquiera una conciencia de su propia importancia no solo para sí mismo sino para toda la sociedad"1.

Se debe, entonces, promover la participación activa de la sociedad como pilar fundamental de la democracia, haciéndose necesario que los jóvenes sean formados en elementos básicos de ciudadanía para que puedan reaccionar de manera asertiva ante las diferentes situaciones que se presentan en su contexto, ya que la participación "no es un evento o una instancia, sino un proceso complejo e integral, donde uno de los objetivos primordiales es aprender a participar, a través de fortalecer el desarrollo de todas las capacidades de las personas para la vida pública desde una ética de lo colectivo, que fortalezca el tejido social”2.

Para reaccionar como es debido, es decir, para participar activamente en la toma de decisiones y generar propuestas para la consolidación de

1 Alvarado, Wilson, Cárdenas, Lizarda y otros. (1999). Lineamentos para una propuesta conceptual y metodológica para la formación en participación ciudadana. Universidad de la Salle. Bogotá D.C., p. 21.

2 Luna, Milton. (Editor) (2013). Participación ciudadana, políticas públicas y educación. en América Latina y Ecuador. Quito: Organización de Estados Iberoamericanos (OEI), p. 68. 
una realidad social dinámica, participativa, justa y responsable, se hace necesaria que la formación ciudadana sea posible de manera permanente y transversal al proceso formativo en las instituciones educativas. En consecuencia, la universidad debe ser parte indiscutible de esta apuesta de nación, en donde cada estudiante universitario, de cualquier disciplina, reconozca la complejidad de las dinámicas de la realidad social, interprete y sea un agente de cambio en su contexto.

La Universidad Nacional Abierta a Distancia - UNAD es un establecimiento público de carácter nacional que tiene como misión "contribuir a la educación para todos a través de la modalidad abierta, a distancia y en ambientes virtuales de aprendizaje en el marco de la sociedad global y del conocimiento, buscando propiciar el desarrollo económico, social y humano sostenible de las comunidades locales, regionales y globales con calidad, eficiencia y equidad social" ${ }^{\prime 2}$.

Su apuesta es impactar las complejas realidades territoriales a través del reconocimiento de los saberes locales y el fortalecimiento de las capacidades endógenas en la búsqueda de consolidar un "despertar ético, político y pedagógico frente a la cuestión social fundamental, para superar las desigualdades y la crisis humanitaria de tal manera que se promueva la reconstrucción de las comunidades humanas a partir de la globalización de la conciencia solidaria y del espíritu comunitario, mediante la educación abierta, a distancia y en ambientes virtuales $(\mathrm{EaD})^{\prime \prime}$.

De este modo, la UNAD asume el compromiso y el reto de construir "alternativas de solución a los problemas que afectan a las comunidades y a la sociedad colombiana, a partir de la responsabilidad ética, política, social, académica y pedagógica", buscando, además que "Ios aprendizajes sean culturalmente pertinentes, socialmente relevantes e intelectualmente significativos" (PAPS: 2011, p. 14). Precisamente, la UNAD tiene como una de sus responsabilidades sustantivas la inclusión, participación y cooperación desde un énfasis territorial que logre responder adecuadamente a las "necesidades fundamentales (axiológicas

3 UNAD. (2011). Proyecto Académico Pedagógico Solidario (PAPS). Bogotá: Universidad Nacional Abierta y a Distancia, p. 36.

4 Ídem, p. 36. 
y existenciales) que las comunidades y grupos humanos presentan para desarrollar su potencial productivo y de aprendizaje autónomo, producir satisfactores y así impulsar la autogestión del desarrollo a escala humana" ${ }^{5}$.

En consecuencia, la UNAD fundamenta su apuesta académica desde los criterios de sistematicidad ${ }^{6}$ y fractalidad ${ }^{7}$, buscando que los lineamientos, naturaleza y misión institucionales permeen los distintos ámbitos de actuación de la institución y guarden una fuerte identidad organizacional que le permita mantener su carácter y afianzar su impronta de cara a los desafíos que le imponen los distintos contextos de actuación local, regional, nacional y global.

La universidad Nacional Abierta y a Distancia cuenta con 7 escuelas de formación, 24 programas profesionales y una amplia oferta postgradual, llegando a todo el país mediante la modalidad de educación virtual, abierta y a distancia. El programa de Psicología, el cual pertenece a la Escuela de Ciencias Sociales, Artes y Humanidades (ECSAH), tiene alrededor de 15.000 estudiantes convirtiéndolo en el programa con mayor cobertura de la UNAD, no sólo en número de beneficiarios sino en lugares alcanzados por la modalidad. En Ibagué se encuentra el Centro de Educación Abierta y a distancia, perteneciente a la Zona Sur, donde el programa de Psicología tiene inscritos 380 estudiantes, posicionándolo como el programa que acoge el mayor número de estudiantes inscritos en la ECSAH del CEAD Ibagué.

La Escuela de Ciencias Sociales, Artes y Humanidades tiene como misión desde los "procesos de formación, investigación y proyección social contribuir a la construcción de sujetos sociales, que con actitud reflexiva, crítica y creativa interpretan la realidad y promuevan la transformación social, con criterio solidario e incluyente" ${ }^{\prime \prime}$. En coherencia, el programa de Psicología de la Universidad se materializa y responde no sólo a los

5 Max-Neef, M. (1986). Desarrollo a escala humana: una opción para el futuro. Santiago de Chile: CEPAUR.

6 La organización es un todo en donde sus diversas unidades misionales y de gestión están correlacionadas y guardan unidad de propósito (PAPS, p.38).

7 La institución asume que la identidad es una constante de las diversas formas de presencia y desarrollo institucional en sus múltiples contextos de actuación (PAPS, p. 38).

8 Extraído de la página https://academia.unad.edu.co/ 
criterios de calidad y pertinencia disciplinar y profesional, permitiéndole al egresado el "adecuado desempeño en diferentes escenarios de actuación, con actitud ética y compromiso social, para aportar al desarrollo humano y comunitario", sino que expone adecuadamente el interés de la UNAD por extender la formación ciudadana y la construcción de sujetos sociales con actitud crítica y creativa.

Surge entonces la necesidad de evidenciar cuál es el aporte que viene realizando la educación superior, en este caso en particular, la Universidad Nacional Abierta y a Distancia - UNAD con su Proyecto Académico Pedagógico Solidario desde su programa de Psicología con sede en la ciudad de Ibagué, para la formación ciudadana de sus educandos, y así determinar si el énfasis que le imprimen a su proyecto universitario es "culturalmente pertinente, socialmente relevante e intelectualmente significativo" (PAPS: 2013, p. 14) con los horizontes de cambio que se vienen generando en Colombia en relación con la convivencia pacífica, la consolidación de escenarios de participación, el empoderamiento ciudadano y un sinnúmero de trasformaciones que se vienen gestando y para lo cual la educación y el sistema educativo deben ser pilar fundamental.

\section{Antecedentes de investigación}

Para el desarrollo de esta investigación se llevó a cabo un rastreo de antecedentes con la intención de propender por una aproximación a un estado del arte que dilucide panoramas en ámbitos internacionales y nacionales en relación con las categorías genéricas que marcan el derrotero de esta apuesta académica e investigativa, es decir, desde la formación ciudadana y la ciudadanía.

María Ros, de la Universidad Complutense de Madrid, y Valdiney Gouveia, de la Universidad Federal de Paraíba, Brasil (2001), en su documento La ciudadanía, referencian un estado del arte sobre dicho concepto donde se reflexiona tanto como derecho institucional, por lo cual es necesario su ejercicio desde el respeto a la ley y a las normas, eje fundamental de la convivencia, pero también desde la educación para la libertad y desde las capacidades humanas. Las autoras indican que su libro es fruto de la colaboración de un grupo de investigadores interesados en el estudio de los valores humanos, que sienten la necesidad de reunir en 
un mismo volumen en castellano algunos de los trabajos más recientes y representativos en el desarrollo teórico y en la investigación sobre el tema. Piensan que de este modo se llenará un vacío importante para el desarrollo de una masa intelectual crítica sobre el papel de las prioridades de los valores en nuestras sociedades y en nuestro comportamiento intra e interpersonal. (Ros María y Gouveia Valdiney, 2001, p. 17). Un aporte del documento es el desarrollo que plantean en cuanto a valores a través de tres etapas: 1) los antecedentes históricos procedentes de la sociología y la psicología; 2) los desarrollos realizados en los años 70; y 3 ) la etapa de los años 80 y 90 . Las autoras argumentan que este desarrollo ha hecho posible que en la actualidad tengamos teorías integradas sobre la estructura de los valores a nivel individual y cultural, que permiten el análisis y la comparación de tipos o síndromes de valores articulados en torno a dimensiones transculturales de comparación". (Ros María y Gouveia Valdiney, 2001, p. 18).

Por otro lado, en cuanto a la reflexión de ciudadanía y los valores que la constituyen resulta preponderante traer a colación el Estudio Mundial de Valores desarrollado por el Grupo Europeo de Estudios sobre Valores EMV, bajo la dirección de Jan Kerkhofs y Ruud de Moor. Dicho estudio de valores se desarrolló en 10 sociedades del occidente de Europa y generó tanto interés que se replicó en 14 países. El objetivo del estudio mundial de valores es observar la evolución que se da en la manera de valorar en diferentes países y regiones del mundo. Observa los cambios políticos y económicos, las normas de la religión, los valores de la familia y explora cómo estos cambios afectan las bases del crecimiento. La utilidad de estos estudios ha crecido tanto que se han convertido en la fuente más completa de información y cobertura de las sociedades del mundo, y las series de tiempo de información generadas se han fortalecido.

Otro estudio en el campo de los imaginarios sociales en ciudadanía se encuentra en España: una línea del "Grupo Compostela de Estudios sobre Imaginarios Sociales" (GCEIS), que en los últimos años se consolida como grupo de investigación y que realiza, también, aportes significativos en América Latina, donde se destaca por la producción intelectual que realiza en torno a la teoría de imaginarios sociales, las actuales perspectivas fenomenológicas y las nuevas técnicas cualitativas de investigación social, para contribuir a la generación de conocimientos para las Ciencias Sociales. 
Así mismo, el Proyecto colectivo de enlace nuevas formas de democracia, nuevas formas de ciudadanía: hacia una propuesta alternativa de la educación ciudadana, (2008), que comprende dos grandes actividades, promover un diálogo internacional en torno al tema del Proyecto y, desarrollar una investigación-acción sobre cultura y competencias ciudadanas de estudiantes pre y universitarios.

En Latinoamérica, en la Universidad de Chile, se encuentra el Programa Ciudadanía, Participación y Políticas Públicas, implementado por el Departamento de Políticas Públicas del Instituto de Asuntos Públicos, que desarrolla investigaciones multidisciplinarias desde una perspectiva académica, y busca contribuir al desarrollo y la consolidación democrática mediante la profundización del conocimiento acerca de las interrelaciones entre ciudadanía, participación social y políticas públicas.

Héctor Luis Lacreu, en su trabajo titulado Formación ciudadana en la universidad, una asignatura pendiente (2013), presentado en el Congreso Latinoamericano de Educación Superior en el siglo xxi, San Luis, 18 al 20 de setiembre de 2003, en San Luis, México; explícita que el desprecio de la sociedad por la política y las instituciones también se hace extensivo, aunque en menor medida, a la institución universitaria. En parte, ello se manifiesta en la falta de comunicación y el desinterés de cada sector respecto de lo que le pasa al otro, así como en la escasa participación de los estudiantes en la defensa de sus propios intereses. Esta situación constituye un círculo vicioso que la universidad debería tomar la iniciativa en destruir, promoviendo una mayor comprensión y compromiso de estudiantes y docentes con los problemas sociales.

En su artículo Formación para la ciudadanía y educación superior (2006), Miquel Martínez Martín aborda cómo la universidad es el lugar en el que se aprende el conjunto de saberes que permitirán al futuro titulado ejercer una profesión o dedicarse al ámbito de la investigación. Sin embargo, no resulta tan obvio que la universidad sea un lugar en el que se aprenda un conjunto de saberes éticos y ciudadanos. En este artículo se sostiene que una de las funciones de la formación universitaria es de carácter ético y que no puede entenderse una formación universitaria de calidad que no incorpore de forma sistemática y rigurosa situaciones de aprendizaje ético y de formación ciudadana. Se identifican tres dimensiones formativas en la función ética de la universidad en la sociedad actual: la 
formación deontológica, relativa al ejercicio de las diferentes profesiones; la formación ciudadana y cívica de sus estudiantes; y, finalmente, la formación humana, personal y social, que contribuya a la optimización ética y moral de las futuras y futuros titulados en tanto que personas.

Por su parte, en conjunto con la Universidad de Santiago de Compostela, en España, la Universidad Nacional del Nordeste, en Argentina, en 2005, bajo la estrategia de la investigación cualitativa, a través de los grupos de discusión, adelantó una investigación liderada por Ana María Pérez y María del Socorro Foio, con el título Ciudadanía: imaginario social y representaciones sociales, con el propósito de avanzar en el proceso de reconstrucción de sentidos del accionar de la población en el espacio público y develar los distintos significados que las nociones de justicia, derechos y ciudadanía adquieren actualmente, en especial, la relación que tiene el papel del Estado como garante del sistema de relaciones sociales.

Entre dichos estudios se encuentra el documento titulado Formación ética, valores y democracia, una educación para la ciudadanía, de Guillermo Hoyos Vásquez (2000), donde se expresa que si no utilizamos la educación para lo que se inventó, es decir, para formar ciudadanos, y si no los formamos con base en principios y valores para la convivencia, nos hemos "rajado" en educación y en pedagogía, así estemos diseñando ya estudios científicos en educación; de igual manera, aporta un marco teórico consolidado, basado en Apel, Habermas, Kohlberg y Cortina.

Otro documento es la investigación de realizada por Diana María Posada, Martha Lorena Salinas Salazar y Luz Estela Isaza Mesa, de la Universidad de Antioquia, sobre "Las representaciones sociales sobre el valor justicia como punto de partida para el fortalecimiento de la convivencia escolar". Este proyecto focaliza su interés en las representaciones sociales que sobre la justicia tienen las niñas y los niños y en el efecto que los modos de funcionamiento de la violencia escolar, familiar y social pueden ejercer sobre ellos.

Una investigación realizada en la Universidad de Antioquia por Carlos Sandoval y María Eugenia Villa, titulada Representaciones Sociales, Expresiones de Participación, Razonamiento Social y Prácticas Educativas Relacionadas con la Formación Ciudadana en el Contexto Universitario: 
Un Análisis del Sentido y Condiciones de Posibilidad de un Proyecto de Formación Ciudadana en la Educación Superior; en la cual, su objetivo es identificar y comprender los nuevos modos de ciudadanía que se pudiesen haber configurado con posterioridad a la expedición de la Constitución de 1991 y que muestren signos de existencia en el período 2007-2009. En dicho trabajo se reseña una investigación que realiza la Asociación Internacional para la Evaluación del Logro Educativo IEA que tiene como propósito "identificar y examinar en un marco comparativo las formas en que los jóvenes se preparan para asumir su papel de ciudadanos en las democracias y en sociedades que aspiran a serlo" (Torres y Pinilla, 2005, p. 50). El trabajo de Sandoval y Villa, como ejercicio investigativo, es un buen referente por el marco teórico que desarrollan y por la propuesta metodológica que integra los enfoques cualitativo y cuantitativo.

Por su parte, Manuel Jair Vega y Luz Helena García, de la Universidad del Norte, llevan a cabo un estudio sobre imaginarios de ciudad con niñas y niños de estrato alto, medio y bajo, entre los 9 y los 11 años, elaborado en la ciudad de Barranquilla durante 2005 que permite ver cómo los niños se ven integrados, como ciudadanos, en su sociedad y descubrir los aspectos que fortalecen o debilitan el interés hacia los asuntos colectivos. Esta investigación se centró en imaginarios porque los autores consideran que la manera como la realidad es pensada tiene consecuencias en la manera como se vive en la sociedad. Los datos se recogieron a través de grupos de discusión y el registro en notas de campo. Se exploraron los imaginarios de ciudadano, poder y participación de niños, con el objeto de comprender cómo estos individuos se integran como ciudadanos en su sociedad y qué aspectos fortalecen o debilitan el interés hacia los asuntos colectivos.

En la tesis de maestría Formación para la ciudadanía desde la universidad: una responsabilidad social, realizada por Adrián Andrés Valencia Giraldo en la Universidad del Tolima en el año 2014 se evidencia cómo la formación para la ciudadanía desde la universidad es uno de los elementos esenciales de la Responsabilidad Social Universitaria (RSU). De igual manera, se muestra la relación existente entre Formación para la Ciudadanía y la Responsabilidad Social Universitaria. Este trabajo defenderá la perspectiva de que la formación para la ciudadanía desde la universidad debe estar dirigida a formar un ciudadano democrático, que se asuma como agente, sujeto de derechos y de responsabilidades; 
un ciudadano con interés por la vida pública, con sensibilidad social, comprometido con la defensa y promoción de los derechos humanos, los valores democráticos y con capacidad de resolver pacíficamente los conflictos. Por su parte, plantea la RSU como el fundamento de toda la acción y la gestión universitaria que toca con la naturaleza misma del ser de la Universidad, ya que todo el proceso educativo debe estar orientado a la formación para la ciudadanía, de la cual se sigue todo sentido de responsabilidad con la sociedad.

\section{Metodología}

La investigación es de enfoque mixto con un alcance descriptivo que tiene como diseño metodológico el estudio de caso. El empleo del método mixto brindó metodológicamente amplias técnicas e instrumentos, los cuales permiten un análisis profundo y con rigor de los datos obtenidos. Es así como, por medio del paradigma mixto se aumenta la validez de los hallazgos en vista de que corrobora por medio de métodos cualitativos y cualitativos los datos arrojados. Lo anterior tiene que ver con que el enfoque mixto, ya que corresponde y es consecuente con la búsqueda de la valoración dada por parte de docentes, estudiantes y egresados en relación con la formación ciudadana que reciben y recibieron en el programa de Psicología de la UNAD.

En ese sentido, se opta por las posibilidades del método mixto, entendidas desde la perspectiva de Hernández Sampieri (2003), dado que "La meta de la investigación mixta no es reemplazar a la investigación cuantitativa ni a la investigación cualitativa, sino utilizar las fortalezas de ambos tipos de indagación, combinándolas y tratando de minimizar sus debilidades potenciales" (p. 532). La investigación mixta desde este trabajo no es entendida como una combinación aparente de los métodos de investigación sino como una perspectiva que sustenta una realidad y un contexto. En este mismo sentido, el planteamiento de Cruz \& Campano (2007) argumenta que "Tradicionalmente que contrarresten los paradigmas cualitativo y cuantitativo como un par de polos opuestos, revelando fortalezas y debilidades de ambos lados. Al contraponer aspectos esenciales como los presupuestos, los objetivos y los métodos." (Cruz \& Campano, 2007, p. 7). Por tanto, se escoge el paradigma mixto por la complejidad de contemplar dos enfoques investigativos en una misma línea de pensamiento. Esto permite analizar los datos por medio 
de múltiples maneras que determinan acercamientos al objeto de estudio desde varias perspectivas. Así, la investigación mixta no es una serie de fragmentos en donde, por un lado se emplea acciones de la investigación cuantitativa, y luego, en otro apartado, se emplean aspectos de la investigación cualitativa. Por el contrario, es una cohesión y una articulación que proponen rutas en las cuales se interpreta la realidad, donde también hay una comprensión y trabajo hermenéutico de los datos numéricos y estadísticos.

El impacto que tiene la investigación mixta acontece porque coloca al investigador como un ente activo dentro de las construcciones de conocimiento. El investigador debe interpretar múltiples esferas que coexisten en un contexto. Tashakkori y Teddlie (2003) plantean que, a través de la investigación mixta, el investigador debe utilizar su capacidad analítica e interpretativa para asociar, correlacionar las variables para objetivar los resultados y transfórmalos en conocimientos. De esta manera, existe una compresión de la realidad y también una demostración con el propósito de dar una mirada holista y no reducir el fenómeno estudiado a una sola forma investigativa.

Ahora bien, el diseño metodológico de estudio de caso fue pertinente, dado que, al buscar valorar concepciones acerca de la formación ciudadana en estudiantes, docentes y egresados del programa de Psicología de la UNAD, lo que se encuentra es múltiple, por lo cual desde el estudio de caso de un grupo determinado, se posibilita dilucidar un panorama más generalizado. Por otro lado, se articula con este proyecto investigativo, ya que "el estudio de caso es un método de investigación cualitativa y empírica orientada a la comprensión en profundidad de un objeto, hecho, proceso o acontecimiento en su contexto natural. Se utiliza tanto en investigaciones propias del paradigma interpretativo como del sociocrítico" (Rovira, Codina, Marcos, y Palma: 2004, p. 11).

De igual manera, se parte de lo expresado por Robert Stake, quien expresa que "de un estudio de caso se espera que abarque la complejidad de un caso particular. Una hoja determinada, incluso un solo palillo, tienen una complejidad única -pero difícilmente nos preocuparán lo suficiente para que los convirtamos en objeto de estudio" (1999, p. 14). Estas palabras corresponden a la descripción y comprensión de concepciones, representaciones, incluso prácticas en torno a la formación ciudadana 
de los estudiantes del programa de Psicología en la Universidad Nacional Abierta y a Distancia, CEAD Ibagué.

El estudio giró en torno a la audiencia foco, integrada por tres grupos de actores sociales, los cuales, de acuerdo con los objetivos específicos planteados inicialmente, contribuyeron al logro del objetivo general en diferentes momentos de la investigación. Los grupos se describen a continuación: - Comunidad estudiantil del programa de Psicología del CEAD Ibagué; - Grupo de cinco (5) docentes del programa de Psicología del CEAD Ibagué, quienes participaron en la formación de los estudiantes de prácticas profesionales. - Grupo de cinco (5) egresados del programa de Psicología del CEAD Ibagué para el grupo focal.

\section{Instrumentos}

De acuerdo con la metodología planteada se optó en un primer momento por llevar a cabo una revisión documental, a partir de la cual se elaboró una encuesta cualitativa, para después terminar de adentrase en la temática a través del grupo focal. La diversidad de instrumentos utilizados tuvo como propósito complejizar la mirada y armonizar las diferentes perspectivas que aportaron al análisis y a la triangulación de información.

\section{Análisis de resultados y discusión}

\section{Análisis de la revisión documental}

En la revisión llevada a cabo de los documentos rectores de la UNAD se evidencia que tanto en la misión como en la visión de la Universidad la formación ciudadana posee un lugar preponderante, aunque algunas veces no se enuncie de manera explícita. Es así como en la UNAD se reconoce el valor de la educación en la sociedad contemporánea no sólo en los aspectos académico y profesional, sino en lo referente a su capacidad crítica, radical y liberadora para la construcción de una sociedad más justa, que busca transformar el contexto social y consolidar imaginarios y valores sociales para afrontar las crecientes problemáticas globales y los incipientes escenarios de un país que tiene la tarea pendiente de vislumbrar nuevos horizontes de la mano de una ciudadanía activa. Estos presupuestos responden también al mandato ciudadano que trasciende 
el ámbito formal de la participación en el ámbito de lo político, que alude solo a la capacidad de elegir y ser elegido, pues ser ciudadano es ir más allá de la esfera del poder, para formarse y participar en todo aquello que lo afecta; es también crear vínculos solidarios y elaborar un ideal democrático que procure el bienestar común.

Ahora bien, en la revisión del PAPS (Proyecto Académico Pedagógico Solidario), de la UNAD, se asume el compromiso y el reto de construir "alternativas de solución a los problemas que afectan a las comunidades y a la sociedad colombiana, a partir de la responsabilidad ética, política, social, académica y pedagógica", buscando además promover escenarios de aprendizajes pertinentes y relevantes. Precisamente, la UNAD tiene como una de sus responsabilidades sustantivas la inclusión, la participación y la cooperación desde un énfasis territorial que logre responder a las "necesidades fundamentales (axiológicas y existenciales) que las comunidades y grupos humanos presentan para desarrollar su potencial productivo y de aprendizaje autónomo, producir satisfactores y así impulsar la autogestión del desarrollo a escala humana".

De aquí la importancia de evidenciar la contribución que viene realizando la Educación Superior, en este caso en particular la Universidad Nacional Abierta y a Distancia (UNAD) con su Proyecto Académico Pedagógico Solidario a través del programa de Psicología con sede en la ciudad de Ibagué, en relación con la formación ciudadana de sus educandos, buscando determinar si el énfasis que la Universidad le otorga a su Proyecto Universitario es "culturalmente pertinente, socialmente relevante e intelectualmente significativo" (p. 14).

Por su parte, para organismos internacionales como la UNESCO, la formación ciudadana debe considerar una serie de atributos y lineamientos, proponiendo de este modo un conjunto de competencias ciudadanas, como son:

(1) el conocimiento y la comprensión de temas y tendencias mundiales específicos, y el conocimiento y el respeto por los valores universales esenciales (la paz y los derechos humanos, la diversidad, la justicia, la democracia, la solidaridad, la no discriminación y la tolerancia); (2) las habilidades cognitivas para un pensamiento crítico, creativo e innovador para la resolución de problemas y la toma de decisiones; (3) las habilidades no cognitivas como la empatía, la apertura hacia experiencias y perspectivas distintas, las habilidades interpersonales y de comunicación, 
y la aptitud para establecer redes e interactuar con personas de diferentes extracciones y orígenes; (4) la capacidad de iniciar y participar en acciones de forma proactiva (UNESCO, 2013).

En ese sentido, y teniendo en cuenta tanto las competencias como los pilares de la educación, el enfoque de formación ciudadana proclamado por la UNESCO vincula las habilidades del siglo XXI y contextualiza sus requerimientos en un mundo globalizado. No obstante, la formación ciudadana, a pesar de que en ocasiones suene como un asunto incipiente y se asuma como una temática novedosa, es una propuesta que ha orientado el espacio educativo y ha sido una constante preocupación de los sistemas escolares a lo largo de su historia.

\section{Análisis de la encuesta}

De acuerdo con la investigación, se aplicó una encuesta de carácter cualitativo a los 18 estudiantes de la audiencia foco del programa de Psicología de la UNAD del CEAD Ibagué para recolectar información que permitiera complementar el análisis en lo referente a las apuestas del diseño institucional alrededor de la formación ciudadana. Dicha encuesta fue enviada a través de un formulario virtual remitido a través de los números telefónicos (Whatsapp) y/o vía correo electrónico institucional de los estudiantes. El instrumento está compuesto por veintiún ítems y tiene como opción la respuesta abierta, el sí y no o la selección múltiple, las cuales están dirigidas a evidenciar las tres dimensiones de las competencias ciudadanas: 1 . Convivencia y paz, de la pregunta seis a la once (6-11), 2. Participación y responsabilidad democrática, de la pregunta doce a la dieciocho (12-17), y por último 3. Pluralidad, identidad y valoración de las diferencias, de la pregunta dieciocho al veintiuno (1821). Es importante aclarar que estas dimensiones no están enunciadas en el instrumento que fue remitido a los estudiantes.

De acuerdo con la información recolectada, se infiere que la mayoría de los estudiantes asocian la formación ciudadana como un tema de responsabilidad y que corresponde a las funciones básicas del sistema educativo, ya sea en la educación básica o profesional. En otras palabras, existe una visión instrumental respecto de la formación ciudadana que le compete solo al ámbito pedagógico, dejando de lado otras instituciones sociales como la familia; además, logra transmitirse y validarse no de forma transversal y generativa, sino de manera coyuntural y a cuentagotas en algunos espacios eventualmente dispuestos para ello. 
Ahora bien, los estudiantes también reconocen que la cultura democrática posee un fuerte vínculo con la educación, expresado en aquello que aprendemos y compartimos con otras personas o elaboramos en relación con el entorno; es decir, hay una constante mediación educativa que trasciende la vida académica para instalarse en lo cotidiano. Asimismo, consideran que la formación ciudadana permite un reconocimiento de la alteridad y la valoración de la diferencia, lo cual le posibilita al estudiante estar rodeado de personas que piensan o actúan diferente $y$, sin importar esa distancia, llegar a unos arreglos mínimos ajustados a los valores socialmente compartidos.

En gran parte de las respuestas obtenidas, los estudiantes reafirman las preguntas anteriores asociadas a los valores, actitudes y maneras de relacionarse con el otro, lo que valida la importancia otorgada a la formación ciudadana. De este modo, se reconoce la necesidad de escuchar lo que el otro tiene que decir frente a diversos temas y aceptar sus posturas de forma respetuosa y creativa.

En cuanto a la pregunta que se refiere al hecho de denunciar cuando se tiene conocimiento de una falta o delito, resulta interesante evidenciar la apatía y rechazo por asumir una responsabilidad civil frente a una trasgresión de la norma, mostrando además una profunda desconfianza y escepticismo frente a la eficiencia y celeridad de las instituciones encargadas de impartir justicia. Esta desconfianza para acudir oportunamente a las autoridades es otra manera de socavar las aspiraciones y retos ciudadanos, pues si el Estado y las instituciones no pueden garantizar el cumplimiento de los derechos y su oportuna exigencia, además de contribuir a la solución de los conflictos y tensiones que afectan la convivencia, terminan por alegar y minar la voluntad de los ciudadanos para acompañar, participar e incidir activamente en las decisiones de carácter vinculante.

Todas estas posturas reflejan el ideal que tienen los estudiantes unadistas sobre la participación ciudadana, refiriéndola solamente a ejercer su derecho al voto, restringiendo el carácter decisorio de los mecanismos democráticos consagrados constitucionalmente pero que, sin embargo, no se trasladan al ámbito práctico. No obstante, algunos encuestados reconocen y valoran mecanismos como la tutela y el derecho a la protesta, permitiendo abrir otra perspectiva y concepción de lo que implica la participación ciudadana. 
Es paradójico, además, constatar que, aunque la mayoría de los encuestados entienden los alcances y potencialidades de participar activamente en grupos de interés, asociaciones, sindicatos o agrupaciones barriales o locales, también reconocen que no participan de manera concreta y decidida de la denominada sociedad civil, presentándose un desfase entre el deber ser ciudadano y la concreción de las capacidades de mediación e interlocución que en el ámbito cotidiano le provee a los ciudadanos.

\section{Análisis grupo focal}

Para el análisis del grupo focal se tuvieran en cuenta los 3 grupos anteriormente conformados, cada uno por 6 docentes, 6 estudiantes y 6 egresados. El grupo focal tuvo como finalidad profundizar sobre información recolectada mediante la encuesta aplicada a 18 estudiantes de prácticas profesionales del programa de Psicología del CEAD Ibagué acerca de la formación ciudadana. Luego de la apertura se plantearon 3 preguntas: 1. ¿Cuáles son las asignaturas que usted considera aportan a la formación ciudadana de los estudiantes del programa de Psicología de la UNAD? 2. ¿Son pertinentes las orientaciones y contenidos que sobre formación en ciudadanía desarrolla el programa de Psicología? 3. ¿El programa de Psicología de la UNAD promueve la formación de ciudadanía? ¿Cómo?.

\section{Análisis grupo focal Estudiantes}

Las afirmaciones y discusiones en el grupo focal conformado por los estudiantes coinciden en gran medida con lo explicitado en la encuesta, puesto que confirman que valores como el respeto, la responsabilidad y la tolerancia son elementos cruciales que debe promover la formación ciudadana para la buena convivencia. Además, reconocen que mecanismos como el voto permiten de forma concreta afianzar los espacios para asumir el compromiso y los valores ciudadanos. Cabe resaltar que desde el programa de Psicología de la UNAD existen algunos lineamientos para contribuir a fortalecer las comunidades a través de proyectos comunitarios y la intervención social, lo que hace inferir que la participación se concretiza en dichos ámbitos desde una apuesta priorizada desde lo ético, más que desde lo político. 
Finalmente, a pesar de la promoción en torno a los valores ciudadanos que abandera el programa de Psicología de la UNAD, los estudiantes consideran que las asignaturas especialmente enfocadas a promover la formación ciudadana poseen algunas falencias y vacíos para abordar problemáticas de carácter tanto coyuntural como estructural que den cuenta de las dinámicas en que se ven involucrados tanto en su desempeño profesional como en su devenir personal. Además, aunque se promueven algunos espacios extracurriculares para tratar temas concernientes a la responsabilidad y competencias ciudadanas, estos son desarrollados eventualmente y no cuentan con una amplia difusión por parte de la Universidad, sin contar el marcado desinterés y apatía de los estudiantes por participar.

\section{Análisis grupo focal Docentes}

Lo hallado en el grupo focal de docentes resalta puntos comunes como la misión institucional y los valores promovidos de la UNAD, relacionados con la solidaridad y la responsabilidad social. Es así como consideran que desde las asignaturas dadas en el programa de Psicología existe una amplia promoción de las competencias ciudadanas; no obstante, coinciden en que es necesario retomar algunas cátedras y espacios institucionales donde la formación ciudadana sea una preocupación central. También asumen que desde la Universidad se deben abanderar actividades y estrategias extracurriculares que promuevan el debate, la construcción de saberes pertinentes y las habilidades ciudadanas de los estudiantes que se requieren para la construcción de un nuevo escenario más democrático y pluralista. En los docentes del programa de Psicología de la UNAD también sigue siendo marcada la concepción de la ciudadanía como una actividad que responde al cumplimiento de valores y normas, que se ejerce y asume desde acciones puntuales como el derecho al voto, que se convierte casi en la única expresión universal de la participación ciudadana.

\section{Análisis grupo focal Egresados}

En lo expresado y discutido por los egresados participantes del grupo focal desde su experiencia, el trabajo de campo en las prácticas profesionales y a partir de su desempeño laboral en proyectos con las comunidades, le asignan un valor central y preponderante a la 
formación ciudadana que les brinda el programa de Psicología de la UNAD, entendiendo que se constituye en una herramienta a la hora de poder abordar problemáticas puntuales, entablar relaciones con grupos vulnerables, buscar soluciones viables a situaciones complejas y entablar un diálogo generativo con todas las partes involucradas en el proceso.

Precisamente, existe una amplia coincidencia tanto de los estudiantes como de los egresados y docentes en el enfoque de formación orientado a valores a partir del direccionamiento institucional de la UNAD, lo que permite evidenciar rasgos coincidentes en la manera en que los participantes entienden, asumen y valoran la formación ciudadana en el ámbito universitario. Por otra parte, los egresados cuando se refieren a la cátedra de ciudadanía como una asignatura relevante en el programa de Psicología concuerdan con lo manifestado por algunos docentes, quienes expresan la necesidad de fortalecer dicha materia, entendiendo que la formación ciudadana permite dilucidar proyectos de nación más incluyentes, participativos y democráticos.

\section{Triangulación de información}

Al llevar a cabo la triangulación de la información a partir de los lineamientos identificados en la revisión documental, los resultados obtenidos en la encuesta cualitativa y las afirmaciones expresadas en el grupo focal, se pueden identificar varias coincidencias entre lo señalado tanto por los egresados como por los estudiantes que cursan los últimos semestres en el programa de psicología de la UNAD, así como de los docentes que orientan dichas asignaturas, quienes coinciden en que la formación ciudadana debe trascender el ámbito legal y formal para instalarse en las prácticas, relaciones y dinámicas cotidianas de la comunidad universitaria, a fin de que se logren concretar definitivamente las apuestas, retos y esperanzas de una sociedad justa, solidaria y abocada a la formación de los valores humanos.

Es así como la información contenida en el PAPS, las directrices del MEN y los documentos orientadores de la UNESCO ratifican lo expresado por Peña (2001) cuando sostiene "que las sociedades democráticas no pueden asentarse solo sobre los derechos e instituciones; dependen también de las cualidades y actitudes de sus miembros: sentimiento de identidad, tolerancia, participación, responsabilidad y su adhesión 
y participación en el sistema político" (p.216). Estos valores permiten que los ciudadanos puedan "aprender a conocer, es decir, adquirir los elementos de la comprensión, aprender a hacer para poder influir sobre el propio entrono, aprender a vivir juntos, para participar y cooperar con los demás en todas las actividades humanas; por último, aprender a ser, un proceso fundamental que recoge los elementos de los tres anteriores. (UNESCO, 1996: 106).

Justamente, la formación ciudadana tiene que ver con los retos actuales que no sólo se refieren a los aspectos académicos y el ámbito profesional, sino que evocan un componente transformador que impacte sobre la construcción social y el ideal humano, que se interese por afrontar las problemáticas globales, de la misma manera que responde a los retos del entorno sociopolítico, cultural y económico.

Frente a ello, valores como el respeto, la responsabilidad y el compromiso son una alternativa viable para la resolución de conflictos que propicien una ciudadanía activa desde un abordaje académico y pedagógico. En los documentos revisados y en las respuestas halladas en la encuesta y en el grupo focal, se expresan como puntos sustantivos de la formación ciudadana, la inclusión, la participación y la cooperación para resolver las tensiones y necesidades de los diferentes grupos humanos con el propósito de desarrollar sus potencialidades. Es así como dichos valores refuerzan una concepción de formación ciudadana en la que:

\footnotetext{
Se distinguen tres elementos que, en conjunto, constituyen su contenido: el elemento civil, compuesto por los derechos necesarios para la libertad individual: libertad de la persona, de expresión, de pensamiento y religión, derecho a la propiedad y a establecer contratos válidos y derecho a la justicia; el elemento político, cuyo contenido es el derecho a participar en el ejercicio del poder político como miembro de un cuerpo investido de autoridad política o como elector de sus miembros (Marshall, 1992: p. 21).
}

En otras palabras, se reproducen los imaginarios sociales y determinantes culturales que orientan los roles dentro de la sociedad, donde aún se encuentra fuertemente marcada la distinción entre los que gobiernan o no; en ese sentido, el ciudadano continúa representando a "alguien que pertenece plenamente a la comunidad (no es un extranjero, ni un mero residente), que tiene en virtud de ello ciertos derechos (y los deberes correspondientes), y que de algún modo toma parte en la vida pública" (Peña, 2001). 
De modo que desde la formación ciudadana es fundamental promover la politización de "la educación en el sentido de trascender el simple entrenamiento, que no excluya las dimensiones éticas que subyacen a la promoción y utilización de los saberes" (Ospina \& Alvarado, 2006) en contextos donde su concreción se hace imprescindible para conectar con otros y así generar espacios de convivencia y encuentro que procuren la garantía de los derechos de todos. De modo que en las instituciones de educación superior se procure entender la formación ciudadana y la construcción de sujetos con un alto compromiso social, con una actitud crítica y una visión creativa, cobijada por "un Estado que goce de su consenso, que respete sus derechos individuales y que le permita desarrollar sin interferencias su propio plan de vida, según sus gustos y capacidad" (Peña, 2001, p. 233).

Ahora bien, en cuanto a lo expuesto y discutido por los participantes del grupo focal y la encuesta, que aportaron diversas perspectivas y puntos de vista, y en relación con lo anterior, se dilucida que la formación ciudadana que se recibe se da de manera implícita, es decir, no hay una cátedra dedicada exclusiva que se dedique a formar ciudadanamente; por el contrario, hay una pregunta y una reflexión por cómo los valores impactan la formación profesional y académica de los estudiantes. En ese sentido, sigue vigente la concepción de ciudadanía anclada a los valores, en cuanto a lo que tiene que ver con una participación donde implique la ocupación de cargos y la participación en grupos sociales destinados a la defensa y garantía de derechos que en ocasiones se vulneran, de manera que, se promueve un acercamiento a la comunidad, a su empoderamiento a través de actividades investigativas donde los valores poseen una preponderancia mayúscula en la conformación colectiva de la sociedad, de manera que se posibilite trascender ello, para consolidar lo que explicita Adela Cortina, quien se refiere a que la Ciudadanía Política y solo ciudadana, "es primeramente una relación política entre individuo y una comunidad política, en virtud de la cual el individuo es miembro de pleno derecho de esa comunidad y le debe lealtad permanente" (2001, p. 39).

\section{Conclusiones}

- De acuerdo con lo expresado por los docentes, egresados y estudiantes vinculados al programa de Psicología de la UNAD en el CEAD de Ibagué, se puede determinar que existe una valoración positiva, 
pertinente y contextual de la formación ciudadana brindada en las diferentes asignaturas destinadas dentro del plan de estudios a dicho objetivo. La valoración significativa se expresa al estar estrechamente vinculados los lineamientos del MEN, los documentos rectores de la UNAD como es el PAPS, el perfil, la misión y las asignaturas del programa de Psicología, así como lo expresado por los distintos participantes en la investigación. Es posible, entonces, establecer diálogos y preocupaciones comunes que, a pesar de situarse en una ciudad intermedia como lbagué, corresponden a inquietudes y procesos coyunturales de los contextos nacional e internacional.

- EI PAPS (Proyecto Académico Pedagógico Solidario) de la UNAD contiene elementos significativos en relación con la formación ciudadana, puesto que recoge los objetivos y apuestas de la institución universitaria frente a los sujetos que busca formar, los valores que pretende inculcar y todas aquellas características que corresponden a la misión y visión de la Universidad Nacional Abierta y a Distancia. De modo que la UNAD asume el compromiso de la formación universitaria en relación con las situaciones problémicas que suceden a diario y que no permiten el desarrollo sostenible del país. Por tal razón, la Universidad tiene como bandera la responsabilidad social, con lo cual tanto lo ético, como lo político y académico se convierten en bastiones para la contribución a la solución de estos problemas. En este sentido, uno de los grandes baluartes de la UNAD es la apuesta en torno a la formación ciudadana.

- Encuanto alasconcepcionesque poseen los/las estudiantes, docentes y egresados sobre la formación ciudadana que imparte el programa de Psicología de la UNAD - CEAD Ibagué, hay puntos de convergencia en relación con los mecanismos concretos desde los cuales ejercer la ciudadanía. En ese sentido, predomina la relación de la ciudadanía con lo ético y moral, es decir, con normativas y valores que contribuyen a una mejor convivencia; así pues, un buen ciudadano es aquel que ejerce su derecho al sufragio y participa al elegir sus gobernantes y líderes. Esta concepción resulta, sin embargo, problemática, puesto que deja de lado la búsqueda por construir un ideal de nación que garantice resolver los conflictos de intereses de manera concertada, fortalecer las instituciones democráticas, garantizar los derechos ciudadanos y responder a las crecientes demandas sociales, así como robustecer la deliberación y participación de todas y todos. 


\section{Referencias Bibliográficas}

Alvarado, W., Cárdenas, L. et al. \& otros. (1999). Lineamentos para una propuesta conceptual y metodológica para la formación en participación ciudadana. Universidad de la Salle. Bogotá, D.C.

Aristóteles. (2018). Ética a Nicómaco. Madrid: Centro de Estudios Políticos y Constitucionales.

Bellei, C. (Coordinador). (2013). Situación educativa de América Latina y el Caribe: Hacia la educación para todos 2015. Santiago. UNESCO.

Cortina, A. (2001) Ciudadanos del mundo. Hacia una teoría de la ciudadanía. Madrid: Alianza Editorial.

Delors, J. (1994). “Los cuatro pilares de la educación”. En: La Educación encierra un tesoro. México: UNESCO.

Farrés, O. (2010). El transfondo económico de la ciudadanía. En Camps, Victoria (coord.), Democracia sin ciudadanos. Madrid: Trotta.

Horrach, J. (2009). Sobre el concepto de ciudadanía: historia y modelos. En: Revista de Filosofia Factótum, 6, 2009.

Marshall, T. (1992). Ciudadanía y clase social. Madrid: Alianza.

Ministerio de Educación Nacional. (1994). Ley 115 de 1994 General de Educación: El Congreso de la República de Colombia. Recuperado de: https://www. mineducacion.gov.co/1621/articles-85906_archivo_pdf.pdf

Nussbaum, M. (2011). Sin fines de lucro. Por qué la democracia necesita de las humanidades. Madrid: Katz Editores.

Peña, J. (2001). La formación histórica de la idea moderna de ciudadanía. Ponencia presentada en el Seminario Historia y Naturaleza de la UNED, Madrid, España.

Peña, J. (2008). Nuevas perspectivas de la ciudadanía. En: Quesada, F. (coord.), Ciudad y ciudadanía Senderos contemporáneos de la filosofía política. Madrid: Ed. Trotta.

UNAD. (2011). Proyecto Académico Pedagógico Solidario (PAPS). Bogotá.

UNESCO. (2013). Educación para la ciudadanía mundial: una perspectiva emergente. Documento final de la consulta técnica sobre educación para la ciudadanía mundial.

UNESCO. (2013). Docentes para la ciudadanía mundial del siglo XXI. Recuperado de: http://www.unesco.org/new/es/education/resources/onlinematerials/single

UNESCO. (2014). Aprendizajes para el ejercicio de la ciudadanía. Apuntes Educación y Desarrollo Post-2015. Oficina de Santiago Oficina Regional de Educación para América Latina y el Caribe. Recuperado de: http:// www.unesco.org/new/fileadmin/MULTIMEDIA/FIELD/Santiago/pdf/ APUNTE07-ESP.pdf

Universidad Nacional Abierta y a Distancia. (2011). Proyecto académico pedagógico solidario (PAPS) versión 3.0. Bogotá D.C., Agosto 4 de 2011. 